\title{
Search for exotic states in ATLAS: pentaquarks
}

\author{
Ivan Yeletskikh, on behalf of the ATLAS Collaboration \\ Joint Institute for Nuclear Research, Dubna \\ E-mail: ivaneleckih@jinr.ru
}

\begin{abstract}
A study of $J / \psi p$ resonances in the $\Lambda_{b} \rightarrow J / \psi p K$ decays with large $m\left(p K^{-}\right)$invariant masses by the ATLAS experiment at the LHC is presented. The analysis is based on a combined sample of $p p$ collision data at centre-of-mass energies $\sqrt{s}=7 \mathrm{TeV}$ and $8 \mathrm{TeV}$ corresponding to integrated luminosities of $4.9 \mathrm{fb}^{-1}$ and $20.6 \mathrm{fb}^{-1}$, respectively. Although the data prefer the model with two or more pentaquark states, the model without pentaquarks is not excluded. The pentaquark masses and widths obtained using the model with two pentaquarks are consistent with those from the $\mathrm{LHCb}$ experiment.
\end{abstract}

18th International Conference on B-Physics at Frontier Machines - Beauty2019-

29 September $/ 4$ October, 2019

Ljubljana, Slovenia 


\section{Introduction}

Baryons composed of four quarks and one antiquark were suggested in Refs. [1,2] as a natural consequence of the theory of quantum chromodynamics (QCD) and named pentaquarks in Ref. [3]. The experimental observation of pentaquark signals has, historically, been rather controversial [4]. In 2015, the LHCb collaboration has observed two $J / \psi p$ resonant structures in the decay $\Lambda_{b}^{0} \rightarrow J / \psi p K^{-1}$ and interpreted the resonances as the $c \bar{c} u u d$ pentaquark states $P_{c}(4380)^{+}$and $P_{c}(4450)^{+}$[5]. From a fit to the data, the mass of $4380 \pm 8 \pm 29 \mathrm{MeV}$ and width of $205 \pm 18 \pm 86 \mathrm{MeV}$ for $P_{c}(4380)^{+}$, and the mass of $4449.8 \pm 1.7 \pm 2.5 \mathrm{MeV}$ and width of $39 \pm 5 \pm 19 \mathrm{MeV}$ for $P_{c}(4450)^{+}$have been obtained (in quoted uncertainties the first is statistical and the second is systematic). The best fit solution has been found for spin-parity $\left(J^{P}\right)$ values $3 / 2^{-}$ and $5 / 2^{+}$for $P_{c}(4380)^{+}$and $P_{c}(4450)^{+}$, respectively. The fractions of the $\Lambda_{b}^{0} \rightarrow J / \psi p K^{-}$decays with the intermediate $P_{c}(4380)^{+}$and $P_{c}(4450)^{+}$production has been found to be $\sim 8 \%$ and $\sim 4 \%$, respectively [6]. Later, the $\mathrm{LHCb}$ collaboration has provided model-independent evidence for the $J / \psi p$ resonance contribution to the $\Lambda_{b}^{0} \rightarrow J / \psi p K^{-}$decays [7]. The LHCb data for the suppressed $\Lambda_{b}^{0} \rightarrow J / \psi p \pi^{-}$decays also appear to be consistent with two pentaquark signals [8]. However, recently the $\mathrm{LHCb}$ has reported [9] that the $P_{c}(4450)^{+}$signal may represent two narrower states, $P_{c}(4440)^{+}$and $P_{c}(4457)^{+}$, and that there is another narrow resonance, $P_{c}(4312)^{+}$.

In this report, largely based on [10], a study of the $J / \psi p$ resonances in the $\Lambda_{b}^{0} \rightarrow J / \psi p K^{-}$ decays [10] by the ATLAS experiment [11] at the LHC is presented. The analysis is based on a combined sample of $p p$ collision data at centre-of-mass energies $\sqrt{s}=7 \mathrm{TeV}$ and $8 \mathrm{TeV}$ corresponding to integrated luminosities of $4.9 \mathrm{fb}^{-1}$ and $20.6 \mathrm{fb}^{-1}$, respectively $[12,13]$. Due to the absence of particle identification, the $\Lambda_{b}^{0}$ decays are reconstructed together with the decays $B^{0} \rightarrow J / \psi K^{+} \pi^{-}, B^{0} \rightarrow J / \psi \pi^{+} \pi^{-}, B_{s}^{0} \rightarrow J / \psi K^{+} K^{-}$and $B_{s}^{0} \rightarrow J / \psi \pi^{-} \pi^{+}$. These decays to $J / \psi$ and two additional hadrons (protons, kaons or pions) have been reconstructed in events with large invariant masses of the two additional hadrons to suppress dominant backgrounds from light $\Lambda^{*}$, $K^{*}$, etc., states. For the $B^{0} \rightarrow J / \psi K^{+} \pi^{-}$decay, there is a potential contribution from the exotic decays $B^{0} \rightarrow Z_{c}(4200)^{-} K^{+} \rightarrow J / \psi \pi^{-} K^{+}$, that have been recently observed by the Belle collaboration [14]. The effects of this potential $Z_{c}(4200)$ contribution are considered as systematic effects in the analysis.

\section{The ATLAS detector, trigger and data samples}

A detailed description of the ATLAS detector can be found elsewhere [11]. A brief outline of the components most relevant to this analysis is given below.

The ATLAS Inner Detector (ID) has full coverage ${ }^{2}$ in $\phi$, covers the pseudorapidity range $|\eta|<$ 2.5 and operates inside an axial magnetic field of $2 \mathrm{~T}$. It consists of a silicon pixel detector (Pixel), a silicon microstrip detector (SemiConductor Tracker, SCT) and a transition radiation tracker (TRT).

\footnotetext{
${ }^{1}$ Hereafter, charge conjugation is implied.

${ }^{2}$ The ATLAS coordinate system is a Cartesian right-handed system, with the coordinate origin at the nominal interaction point. The anti-clockwise beam direction defines the positive $z$-axis, with the $x$-axis pointing to the centre of the LHC ring. Polar $(\theta)$ and azimuthal $(\phi)$ angles are measured with respect to this reference system. The pseudorapidity is defined as $\eta=-\ln \tan (\theta / 2)$.
} 
The inner-detector barrel (end-cap) parts consist of $3(2 \times 3)$ Pixel layers, $4(2 \times 9)$ double-layers of single-sided SCT strips and $73(2 \times 160)$ layers of TRT straws.

The ATLAS Muon Spectrometer (MS) covers the pseudorapidity range $|\eta|<2.7$. It consists of four detector technologies and a large toroidal magnet system generating an average field of $0.5 \mathrm{~T}$ in the barrel region $(|\eta|<1.05)$ and $1 \mathrm{~T}$ in the end-cap region $(1.05<|\eta|<2.7)$.

The ATLAS detector has a three-level trigger system [15, 16]: the hardware-based Level1 system and the two-stage High Level Trigger (HLT). For this measurement, triggers based on single-muon, dimuon and three-muon signatures are used. The third muon can be produced in $\Lambda_{b}^{0}$ signal events with semileptonic decays of another $b$ quark. At Level-1, muon triggers search for patterns of MS hits corresponding to muons passing various $p_{\mathrm{T}}$ thresholds. Since the rate from the low- $p_{\mathrm{T}}$ muon triggers was too high, prescale factors were applied to reduce their output rates. The majority of events are collected by dimuon triggers with $p_{\mathrm{T}}$ thresholds of $4 \mathrm{GeV}$ for both muons, $4 \mathrm{GeV}$ and $6 \mathrm{GeV}$ for two muons, and $6 \mathrm{GeV}$ for both muons. Single-muon triggers increase the acceptance for asymmetric $J / \psi$ decays where one muon has $p_{\mathrm{T}}>6 \mathrm{GeV}$. Three-muon triggers had a $p_{\mathrm{T}}$ threshold of $4 \mathrm{GeV}$, thus enhancing the acceptance for periods when $p_{\mathrm{T}}$ threshold for at least one muon in the dimuon triggers was $6 \mathrm{GeV}$. At HLT, the dimuon triggers, used in this analysis, require muons with opposite charges and dimuon mass in the range $2.5<m\left(\mu^{+} \mu^{-}\right)<4.3 \mathrm{GeV}$.

This analysis is based on a combined sample of proton-proton collision data collected by the ATLAS detector at the LHC at center-of-mass energies $\sqrt{s}=7 \mathrm{TeV}$ and $8 \mathrm{TeV}$ corresponding to integrated luminosities of $4.9 \mathrm{fb}^{-1}$ and $20.6 \mathrm{fb}^{-1}$, respectively.

The event sample is processed using the standard offline ATLAS detector calibration and event reconstruction code. Only events with at least four reconstructed tracks and at least one reconstructed primary vertex formed by three or more tracks are kept for further analysis.

\section{Event and $B$-hadron candidate selection}

Events are required to contain at least two muons identified by the MS with tracks reconstructed in the ID. The muon track parameters are taken from the ID measurement alone, since the MS does not significantly improve the precision in the momentum range relevant for the $J / \psi$ measurements presented here. To ensure accurate measurements, each muon track must contain at least six SCT hits and at least one Pixel hit. Muon candidates satisfying these criteria are required to have opposite charges and a successful fit to a common vertex with $\chi^{2} / N_{\text {dof }}<10$. The muon transverse momenta and pseudorapidities are required to be in the ranges:

$$
p_{\mathrm{T}}\left(\mu^{ \pm}\right)>4 \mathrm{GeV},\left|\eta\left(\mu^{ \pm}\right)\right|<2.3,
$$

to ensure high trigger and reconstruction efficiency. Events with $m\left(\mu^{+} \mu^{-}\right)$values within $\pm 290 \mathrm{MeV}$ intervals around the $J / \psi$ world average mass [17] are used to search for $B$-hadron $\left(H_{b}\right)$ candidates. All $J / \psi$ candidates are used in each event to form $H_{b}$ candidates.

Tracks of the selected $J / \psi$ and two additional tracks from charged particles with $|\eta|<2.5$ are refitted to a common vertex with the dimuon mass constrained to the world average mass of $J / \psi$. Both combinations with opposite charges of two hadron tracks, as expected for the neutral B-hadron decays, and combinations with the same charges (wrong-charge combinations) are kept at this stage. 
The $B$-hadron candidate momentum $\left(H_{b}=\Lambda_{b}, B^{0}\right.$ or $B_{s}$ depending on the hadron mass assignments) is determined from the sum of the reconstructed $J / \psi$ momentum and hadron track pair momenta.

The primary vertex candidate with the smallest value of the three-dimensional impact parameter of the $H_{b}$ candidate is selected as the actual primary vertex. To improve reconstruction resolution of the $J / \psi$ and two additional hadrons momenta, the $H_{b}$ vertex fit is repeated with the requirement on the $H_{b}$ candidate momentum to point to the primary vertex. The track parameters from the former fit are used in the invariant mass calculations.

To suppress combinatorial backgrounds the following requirements are used:

- $\chi^{2}\left(H_{b}\right) / N_{\text {dof }}<2$, where $\chi^{2}$ is the quality of the fit to the $H_{b}$ topology with $N_{\text {dof }}=8$.

- $L_{\mathrm{xy}}\left(H_{b}\right)>0.7 \mathrm{~mm}$, where $L_{\mathrm{xy}}\left(H_{b}\right)$ is the transverse decay length of the $H_{b}$ vertex measured from the primary vertex.

- $p_{\mathrm{T}}\left(H_{b}\right) / \sum p_{\mathrm{T}}($ track $)>0.2$, where the sum in the denominator is taken over all tracks originating from the primary vertex (tracks of the $H_{b}$ candidate are included in the sum). The requirement removes a sizeable fraction of combinatorial background while having a smaller effect on the signal due to the characteristic hard fragmentation of $b$ quarks.

- $p_{\mathrm{T}}(p)>2.5 \mathrm{GeV}$ and $p_{\mathrm{T}}\left(K^{-}\right)>1.8 \mathrm{GeV}$, assuming proton and kaon masses for the additional tracks in turn.

- $\cos \theta_{P_{c}}<0.5$, where $\theta_{P_{c}}$ is the angle between $J / \psi$ momentum in the $P_{c}$ candidate rest frame and $P_{c}$ candidate momentum in $\Lambda_{b}$ candidate rest frame;

- $\cos \theta_{\Lambda_{b}}<0.8$, where $\theta_{\Lambda_{b}}$ is the angle between $P_{c}$ candidate momentum and $\Lambda_{b}$ candidate momentum in laboratory frame;

- $\left|\cos \theta_{\Lambda^{*}}\right|<0.85$,

where $\theta_{\Lambda^{*}}$ is the angle between kaon momentum in $\Lambda^{*} \rightarrow p K$ candidate rest frame and $\Lambda^{*}$ candidate momentum in $\Lambda_{b}$ candidate rest frame.

Only $H_{b}$ candidates with

$$
p_{\mathrm{T}}\left(H_{b}\right)>12 \mathrm{GeV},\left|\eta\left(H_{b}\right)\right|<2.1
$$

are retained for further analysis.

In the absence of particle identification, the selected sample is dominated by combinatorial background and $H_{b}$ decays to $J / \psi$ and a light neutral meson decaying to two hadrons $\left(\Lambda_{b} \rightarrow\right.$ $\left.J / \psi \Lambda^{*}, B^{0} \rightarrow J / \psi K^{*}, B_{s}^{0} \rightarrow J / \psi \phi / f_{2}\right)$. These contributions are concentrated at small values of the invariant mass of the two hadrons. To suppress the backgrounds, the requirement $M(K \pi)>$ $1.55 \mathrm{GeV}$ is applied to both mass hypothesis combinations of the hadron tracks. The requirement $M(K \pi)>1.55 \mathrm{GeV}$ selects candidates with $M(p K)>2.0 \mathrm{GeV}$.

In rare events with more than one selected $H_{b}$ candidate all candidates are considered. Number of such candidates is $<1 \%$ relative to the total number of $H_{b}$ selected. 


\section{Signal modelling}

Modelling of the signal and dominant backgrounds is done using PYTHIA 8.1 [18] event generator using the so-called "phase space" model for the decays. This simplified model simulates $B$-hadron production, three-particle phase space decays $B \rightarrow J / \psi h h$, and $J / \psi$ decays into muonantimuon pairs. The phase space MC for the $\Lambda_{b} \rightarrow J / \psi p K, B^{0} \rightarrow J / \psi K \pi, B_{s}^{0} \rightarrow J / \psi K K$ processes are produced. The generation is based on leading-order matrix elements for all $2 \rightarrow 2$ QCD processes. Initial- and final-state parton showering is used to simulate higher-order processes. The generated events with the muons having transverse momenta above $3.5 \mathrm{GeV}$ and pseudorapidities within \pm 2.5 are passed through a simulation of the detector using the ATLAS simulation [19] based on GEANT4 $[20,21]$ and processed with the same reconstruction program as used for the data. A simulation of the triggers and their prescale factors is applied to the MC samples.

At the stage of the model fits to data, each generated phase space MC event is weighted with the analytically derived decay matrix element [22, 23, 24].

The matrix element is a coherent sum of helicity amplitudes of all resonant contributions to the $B$-hadron decays, square-averaged over external particles $\left(\Lambda_{b}, p\right.$, muons) polarisations. It includes known and (optionally) exotic resonant terms and accounts for interference effects and spin effects in all decays in the decay chain. Free parameters of the model - a set of decay coupling constants as well as masses and widths of new states - are defined from the fits to data.

The well established resonances contributing to $\Lambda_{b}^{0} \rightarrow J / \psi p K^{-}$decays, in the kinematic region of the selected events, are $\Lambda^{*}(1800), \Lambda^{*}(1810), \Lambda^{*}(1890), \Lambda^{*}(2100)$ and $\Lambda^{*}(2110)$. In the hypothesis of two pentaquark signals, the $\Lambda^{*}$ contributions are summed with two pentaquark $\left(P_{c}(4380)\right.$ and $P_{c}(4450)$ ) contributions. Only the lowest orbital momenta between the $\Lambda^{*}$ and $P_{c}$ decay products are considered in the default model.

The default model for $B^{0}$ decays does not involve any exotic contributions. The possible effect of contribution from the exotic $Z_{c}(4200)$ state is considered as a source of systematic uncertainties. For the $B^{0} \rightarrow J / \psi \pi^{-} K^{+}$decays, the contributions from resonances $K^{*}(1410), K_{0}^{*}(1430)$, $K_{2}^{*}(1430), K^{*}(1680), K^{*}(1780), K^{*}(1950) K^{*}(1980)$ and $K^{*}(2045)$ are involved in the model. For the $B_{s}^{0} \rightarrow J / \psi K^{-} K^{+}$decays, the contributions from resonances $\phi(1680), f_{2}(1525), f_{2}(1640)$, $f_{2}(1750)$ and $f_{2}(1950)$ are involved. All possible orbital momenta between the $B^{0}$ and $B_{s}^{0}$ decay products are considered in the default model.

The set of suppressed decays $B^{0} \rightarrow J / \psi \pi^{-} \pi^{+}$(via intermediate $f_{2}(1270), \rho(1450), \rho(1700)$ and non-resonant phase space decays) and $B_{s}^{0} \rightarrow J / \psi \pi^{-} \pi^{+}$(via intermediate $f_{0}(1370), f_{0}(1500)$, $f_{0}(1790)$ and non-resonant phase space decays) are simulated via dedicated MC samples. The analytical matrix-element modelling is not performed for these decays since their contributions to the kinematic region under interest are marginal.

\section{Fit procedure}

To reduce the combinatorial background effects the fits are performed after subtracting the pure background contribution from combinations with the same charges of two hadron tracks from the contribution of combinations with opposite hadron charges.

All distributions in the paper are shown after this subtraction procedure is applied. 
Due to the absence of hadron identification, the nominal proton, kaon or pion mass [17] can be assigned to each selected additional hadron track. The selected events contain contributions from different $H_{b}$ decays, listed in the previous section 4 . To have credible sensitivity to the different processes, multi-dimensional fits of our model to selected data are performed. Different dimensions here correspond to different hadron mass assignments. Processes displayed with their correct hadron mass assignment are called "direct signals", while processes shown with the wrong mass assignment are "reflected signals".

The following kinematic regions are considered:

- the $\Lambda_{b}$ signal region (SR) defined by the range $5.59 \mathrm{GeV}<m\left(J / \psi, h_{1}=p, h_{2}=K\right)<5.65$ $\mathrm{GeV}$;

- the $B^{0}$ control region (CR) defined by the range $5.25 \mathrm{GeV}<m\left(J / \psi, h_{1}=K, h_{2}=\pi\right)<5.31$ $\mathrm{GeV}$, united with the range $5.25 \mathrm{GeV}<m\left(J / \psi, h_{1}=\pi, h_{2}=K\right)<5.31 \mathrm{GeV}$ with swapped mass hypothesis;

- the $B_{s}^{0}$ control region defined by the range $5.337 \mathrm{GeV}<m\left(J / \psi, h_{1}=K, h_{2}=K\right)<5.397$ $\mathrm{GeV}$.

The contribution from $\Lambda_{b}^{0}$ decays to CRs is small, while $B^{0}$ meson decays represent the largest background in all kinematic regions.

Modelling of contributions from the $H_{b} \rightarrow J / \psi h h$ decays is described in previous section 4 . For the $m(J / \psi h h)$ combinatorial background assessments, functional forms described below are used. Shapes of the combinatorial backgrounds in the SR and CRs are obtained from the events in the lower mass region $(5.35<m(J / \psi p K)<5.45 \mathrm{GeV})$ after rescaling their hadron track momenta. This region is selected as it is dominated by the combinatorial background contribution. Normalisation of the combinatorial backgrounds in the SR and CRs are determined by the $m(J / \psi h h)$ fits.

The $J / \psi$ and additional hadron momenta for each event from the SR are scaled by a small factor which changes the reconstructed $m(J / \psi p K)$ to the nominal $\Lambda_{b}^{0}$ mass [17]. The scaling is performed in the $\Lambda_{b}^{0}$-candidate centre-of-mass system. It improves the $m(J / \psi p)$ resolution from $\sim 9.6 \mathrm{MeV}$ to $\sim 8.4 \mathrm{MeV}$.

Due to complexity of decay model, the fit procedure is iterative. Each iteration includes four steps. The parameters values obtained in each step are used in the following step. After the fourth step, the new iteration starts with step one. The steps are:

- The first step is the so-called "global" fit, which operates over the following distributions:

- 2D $m\left(J / \psi, h_{1}=K, h_{2}=\pi\right)$ versus $m\left(J / \psi, h_{1}=\pi, h_{2}=K\right)$ distribution using the following functional form for the 2D combinatorial background:

$$
\left|x-p_{0}\right|^{p_{1}} \exp \left(p_{2}+p_{3} x\right) \exp \left[-\frac{y-\left(p_{4}+p_{5} x\right)}{p_{6}+p_{7} x}\right]^{2},
$$

where $x=m\left(J / \psi, h_{1}=K, h_{2}=\pi\right), y=m\left(J / \psi, h_{1}=\pi, h_{2}=K\right)$ and $p_{0}-p_{7}$ are free parameters of the fit;

- 2D $m\left(J / \psi, h_{1}=K\right)$ versus $m\left(J / \psi, h_{2}=\pi\right)$ united with $m\left(J / \psi, h_{1}=\pi\right)$ versus $m\left(J / \psi, h_{2}=\right.$ $K)$ distribution in the $B^{0} \mathrm{CR}$; 
- 1D $m\left(h_{1}=\pi, h_{2}=K\right)$ distribution in the $B^{0} \mathrm{CR}$;

- 1D $m\left(h_{1}=K, h_{2}=\pi\right)$ distribution in the $B^{0} \mathrm{CR}$;

- 2D $m\left(J / \psi, h_{1}=K, h_{2}=K\right)$ versus $m\left(J / \psi, h_{1}=\pi, h_{2}=\pi\right)$ distribution using the following functional form for the $2 \mathrm{D}$ combinatorial background:

$$
\left|x-p_{0}\right|^{p_{1}} \exp \left(p_{2}+p_{3} x+p_{4} x^{2}\right) \exp \left[-\frac{y-\left(p_{5}+p_{6} x\right)}{p_{7}+p_{8} x}\right]^{2}
$$

where $x=m\left(J / \psi, h_{1}=K, h_{2}=K\right), y=m\left(J / \psi, h_{1}=\pi, h_{2}=\pi\right)$ and $p_{0}-p_{8}$ are free parameters of the fit;

- 2D $m\left(J / \psi, h_{1}=K\right)$ versus $m\left(J / \psi, h_{2}=K\right)$ distribution in the $B_{s}^{0} \mathrm{CR}$;

- $1 \mathrm{D} m\left(h_{1}=K, h_{2}=K\right)$ distribution in the $B_{s}^{0} \mathrm{CR}$.

The fit function in the "global" fit is built as a sum of the binned log likelihoods calculated for each of the kinematic distributions involved. This fit aims at obtaining parameters of the $B_{0}$ and $B_{s}$ modelled backgrounds to be used in subsequent fits. Parameters of the $\Lambda_{b}$ decays are fixed in the "global" fit to their values, defined at previous iteration.

- The second step is the $\chi^{2} m\left(J / \psi, h_{1}=p, h_{2}=K\right)$ fit. The $B^{0}, B_{s}^{0}$ and $\Lambda_{b}$ decay parameters are fixed in this fit. Free parameters are the total number of the $\Lambda_{b}$ decays, number of the combined $B^{0}$ and $B_{s}^{0}$ decays, as well as the combinatorial background parameters.

- The third step is the fit of the following distributions (build as a sum of their binned log likelihoods):

- 2D $m\left(J / \psi, h_{1}=p\right)$ versus $m\left(J / \psi, h_{2}=K\right)$ distribution in the SR;

- 1D $m\left(h_{1}=p, h_{2}=K\right)$ distribution in the SR.

The purpose of this fit is to obtain the decay constants of the $\Lambda_{b}$ decays.

- The fourth step is the $\chi^{2} m\left(J / \psi, h_{1}=p\right)$ fit in the SR. The pentaquark masses, widths, amplitudes and the relative phase between pentaquark amplitudes $(\Delta \phi)$ are free parameters in this fit.

The iterative procedure is considered as converged if there are no sizeable changes of the fit parameters obtained in the current iteration with respect to those from the previous iteration.

\section{Pentaquark parameters}

The final results are obtained from the $\chi^{2}$ fits of the $m\left(J / \psi p K^{-}\right)$(step 2) and $m(J / \psi p)$ (step 4) distributions after converging of the iterative procedure (4 iterations over all four steps).

Figure 1 shows the $m\left(J / \psi p K^{-}\right)$distribution for all selected $\Lambda_{b}$ candidates. The fit results are shown by the colour-coded histograms. SR is labeled by vertical dashed lines. Events from the SR are used at steps three and four of the fit (ref. Section 5). 


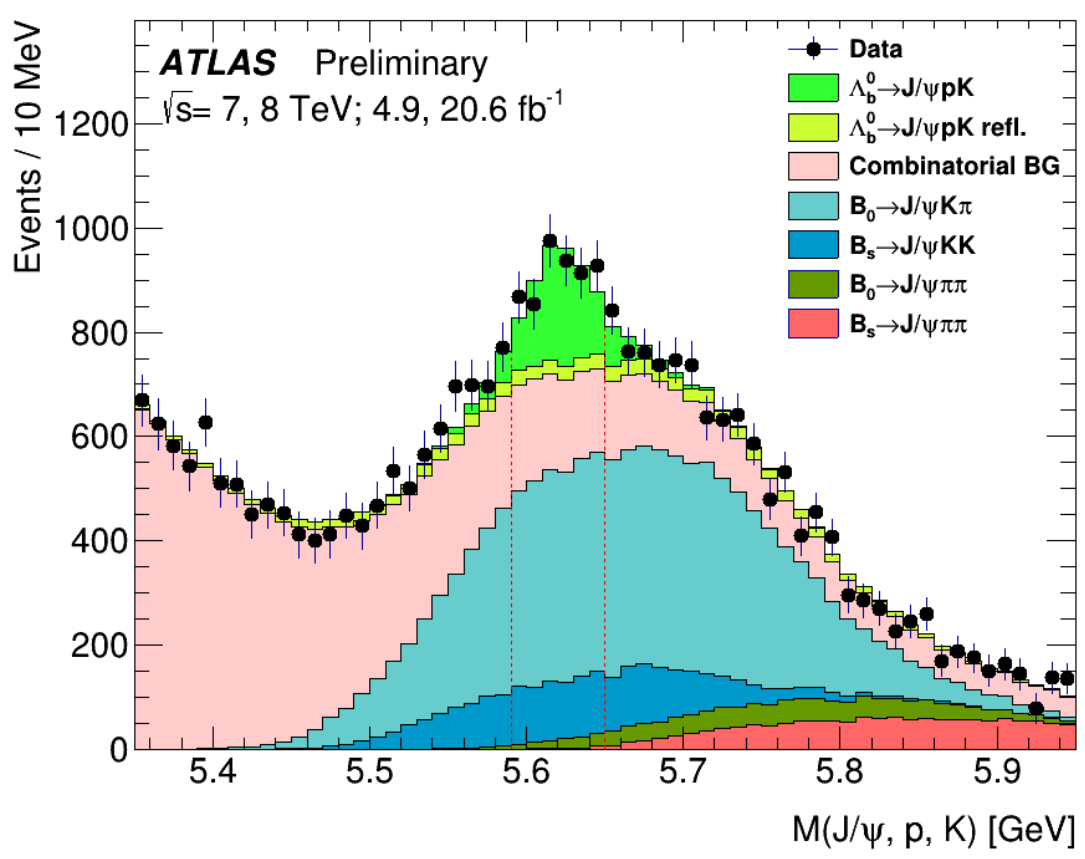

Figure 1: The invariant mass distribution $m\left(J / \psi p K^{-}\right)$for all selected $\Lambda_{b}$ candidates [10]. The results of the iterative fit procedure are shown. Red dashed lines label the signal region: $5.59 \mathrm{GeV}<m(J / \psi, p, K)<$ $5.65 \mathrm{GeV}$.

The combinatorial background distribution is described by the exponential function

$$
\exp \left(p_{0}+p_{1} x\right)
$$

where $x=m(J / \psi p K), p_{0}$ and $p_{1}$ are free parameters of the fit.

The number of $\Lambda_{b}^{0} \rightarrow J / \psi p K^{-}$decays, yielded by the fit, is $2270 \pm 300$, while numbers of other $H_{b}$ decays are $N\left(B^{0} \rightarrow J / \psi K^{+} \pi^{-}\right) \approx 10770, N\left(B_{s}^{0} \rightarrow J / \psi K^{+} K^{-}\right) \approx 2290, N\left(B^{0} \rightarrow J / \psi \pi^{+} \pi^{-}\right) \approx$ 1070 and $N\left(B_{s}^{0} \rightarrow J / \psi \pi^{+} \pi^{-}\right) \approx 1390$. In the signal region, the number of $\Lambda_{b}^{0} \rightarrow J / \psi p K^{-}$decays is $1010 \pm 140$ with correct mass assignments to the final hadrons and $160 \pm 20$ with swapped mass assignments.

The distribution $m(J / \psi p)$ is analysed in the signal region, $5.59<m\left(J / \psi, h_{1}=p, h_{2}=K\right)<$ $5.65 \mathrm{GeV}$. The few first and the few last bins of the distribution are merged together to have enough entries in each bin for the reliable $\chi^{2}$ calculation. Both the data and MC uncertainties are propagated to the fit taking into account double entries from both the "direct signals" and the corresponding "reflected signals", and all weights used in the analysis. 

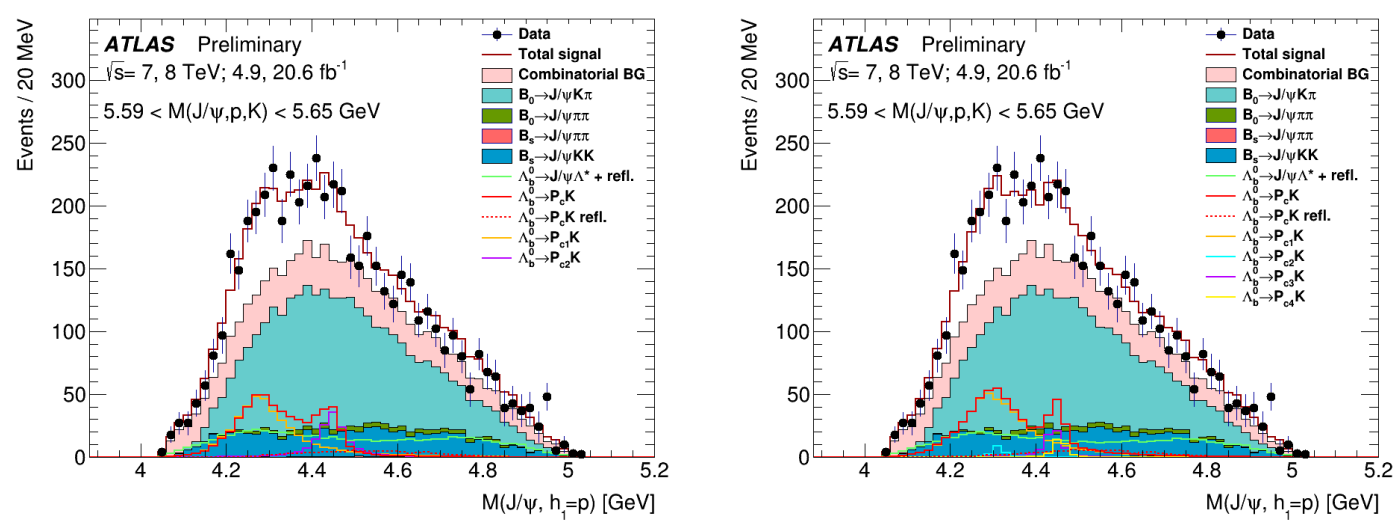

Figure 2: The $\chi^{2}$ fits of the $m(J / \psi p)$ distribution in the signal region [10]. Left: fit with the hypothesis of two pentaquarks $P_{c 1}$ and $P_{c 2}$ with spin-parity $3 / 2^{-}$and $5 / 2^{+}$, respectively. Right: fit with the hypothesis of four pentaquarks $\left(P_{c 1}, P_{c 2}, P_{c 3}, P_{c 4}\right)$.

Figure 2 (left) shows results of the $\chi^{2}$ fits of the $m(J / \psi p)$ distribution for the hypothesis with two pentaquarks with spin-parity $3 / 2^{-}$and $5 / 2^{+}$for lighter and heavier pentaquark candidates, respectively. The data description by this hypothesis is $\operatorname{good}\left(\chi^{2} / N_{\mathrm{dof}}=37.1 / 39\right)$. The narrow pentaquarks reported recently by LHCb cannot be distinguished in the distribution due to the smaller number of signal candidates and the $m(J / \psi p)$ resolution achieved. To verify whether the data are compatible with the LHCb observations, the fit with four pentaquarks is performed with masses, widths and relative yields of the narrow pentaquarks fixed to the LHCb values [9]. The data description by this fit shown in Figure 2 (right) is also good $\left(\chi^{2} / N_{\text {dof }}=37.1 / 42\right)$.
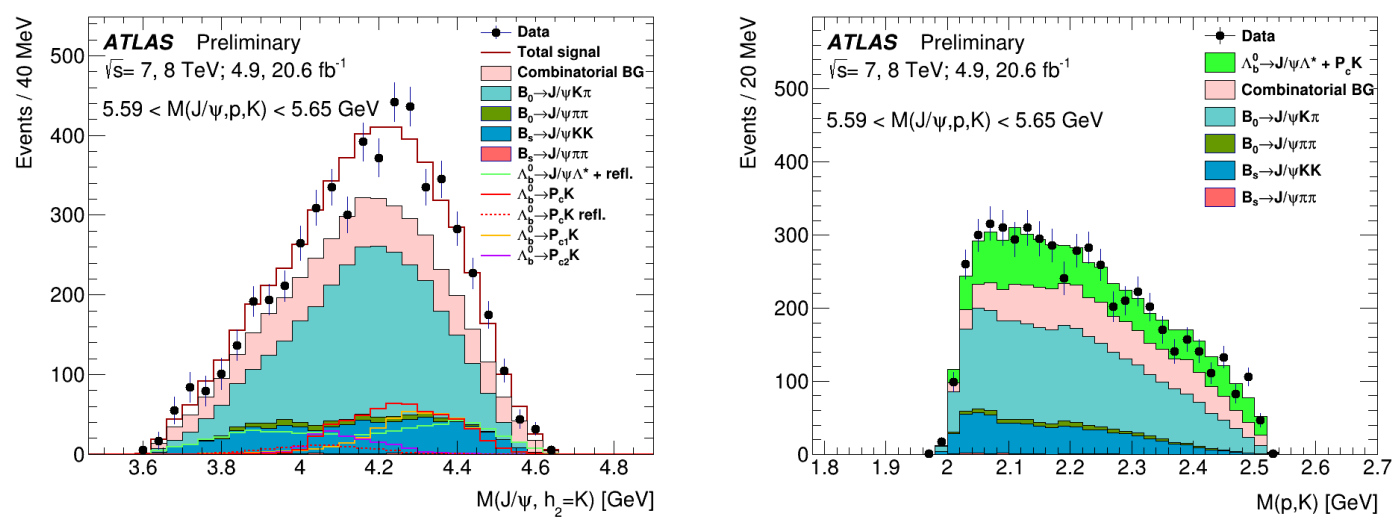

Figure 3: The invariant mass distributions $m(J / \psi K)$ (left) and $m(p K)$ (right) in the signal region overlaid with projections of the fit results for the hypothesis with two pentaquarks with spin-parity $3 / 2^{-}$and $5 / 2^{+}$ for lighter and heavier pentaquark candidates, respectively [10].

Figure 3 shows $m(J / \psi K)$ and $m(p K)$ distributions in the SR overlayed with projections of the central fit results. The model with two pentaquark states describes all these distributions well.

Table 1 summarizes parameters of the $\Lambda_{b}^{0} \rightarrow P_{c}^{+} K^{-}$decays derived from the signal fit. All parameters are shown with their statistical and systematic uncertainties. The statistical uncertainties 


\begin{tabular}{c|c|c}
\hline Parameter & Value & LHCb value [5] \\
\hline \hline$N\left(P_{c 1}\right)$ & $400_{-140}^{+130}(\text { stat })_{-100}^{+110}($ syst $)$ & - \\
$N\left(P_{c 2}\right)$ & $150_{-100}^{+170}(\text { stat })_{-90}^{+50}($ syst $)$ & - \\
$N\left(P_{c 1}+P_{c 2}\right)$ & $540_{-70}^{+80}(\text { stat })_{-80}^{+70}($ syst $)$ & - \\
$\Delta \phi$ & $2.8_{-1.6}^{+1.0}(\text { stat })_{-0.1}^{+0.2}$ (syst $) \mathrm{rad}$ & - \\
$m\left(P_{c 1}\right)$ & $4282_{-26}^{+33}(\text { stat })_{-7}^{+28}($ syst $) \mathrm{MeV}$ & $4380 \pm 8 \pm 29 \mathrm{MeV}$ \\
$\Gamma\left(P_{c 1}\right)$ & $140_{-50}^{+77}(\text { stat })_{-33}^{+41}$ (syst $) \mathrm{MeV}$ & $205 \pm 18 \pm 86 \mathrm{MeV}$ \\
$m\left(P_{c 2}\right)$ & $4449_{-29}^{+20}(\text { stat })_{-10}^{+18}$ (syst $) \mathrm{MeV}$ & $4449.8 \pm 1.7 \pm 2.5 \mathrm{MeV}$ \\
$\Gamma\left(P_{c 2}\right)$ & $51_{-48}^{+59}$ (stat $)_{-46}^{+14}$ (syst) $\mathrm{MeV}$ & $39 \pm 5 \pm 19 \mathrm{MeV}$ \\
\hline
\end{tabular}

Table 1: Parameters of the $\Lambda_{b}^{0} \rightarrow P_{c}^{+} K^{-}$decays derived from the fit (see text) for the hypothesis with two pentaquarks: the pentaquark yields under assumption of no interference effects $\left(N\left(P_{c 1}\right)\right.$ and $\left.N\left(P_{c 2}\right)\right)$, the yield of a sum of two pentaquarks $\left(N\left(P_{c 1}+P_{c 2}\right)\right)$, the relative phase between pentaquark amplitudes $(\Delta \phi)$, and the pentaquark masses and widths [10].

are calculated by means of scanning over the values of each parameter and repeating fits with other parameters kept free; the positive and negative uncertainties correspond to $\chi^{2}$ increase by 1.0 . The pentaquark masses and widths obtained are are consistent with the LHCb results [5] within uncertainties. The fit with the two pentaquark masses and widths fixed to the LHCb values [5] yields $\chi^{2} / N_{\text {dof }}=49.0 / 43$ (with corresponding $p$-value $\left.=24.5 \%\right)^{3}$.

\section{Assessment of systematic uncertainties}

The following groups of systematic uncertainty sources are considered:

- The uncertainty in the number of the $\Lambda_{b}^{0} \rightarrow J / \psi p K^{-}$decays in SR. This is calculated by adding in quadrature the statistical uncertainty of the number (Section 6) and its systematic uncertainty, obtained by variations of the functional form, Eq. (6.1), used for the combinatorial background description and by changing the $m\left(J / \psi p K^{-}\right)$mass range used in the fit at step 2 .

- The uncertainty of the pentaquark modelling. This is determined by comparing the results of the central analysis with those obtained using the following modifications:

- using alternative spin-parity hypotheses, $\left(3 / 2^{-}, 5 / 2^{+}\right)$and $\left(5 / 2^{+}, 3 / 2^{-}\right)$, for two pentaquarks [5];

- using the $P_{c}$ decay model with all possible orbital momenta between their decay products [22, 23, 24];

\footnotetext{
${ }^{3}$ All $p$-values assessments are made in the assumption of the central chi-square distribution
} 
- using the model with four pentaquarks [9].

- The uncertainty of the non-pentaquark $\Lambda_{b}^{0} \rightarrow J / \psi p K^{-}$decay modelling. This is estimated by comparing the results of the central analysis with those obtained using the following modifications:

- using the extended $\Lambda_{b}^{0} \rightarrow J / \psi p K^{-}$decay model with two lowest orbital momenta between the decay products of $\Lambda^{*}(1800), \Lambda^{*}(1810)$ and $\Lambda^{*}(1890)$;

- including the $\Lambda^{*}(2350)$ contribution to the model;

- adding the non-resonant component to the $\Lambda_{b}^{0} \rightarrow J / \psi p K^{-}$decay model either as a wide resonance [5] or as a phase-space decay contribution;

- varying the $\Lambda^{* 0}$ masses and widths within their uncertainties [17].

- The uncertainty of the combinatorial background description for distributions in the $\Lambda_{b}^{0} \mathrm{SR}$. It is determined by varying the maximal allowed momentum corrections and by using different corrections for the proton and kaon momenta.

- The uncertainty of the $B$ meson background description. This is estimated by comparing the results of the central analysis with those obtained using the following modifications:

- using the model including $Z_{c}(4200)$ state. The model including $Z_{c}(4200)$ state and with only the lowest orbital momenta between the $B^{0}$ and $B_{s}^{0}$ decay products is also considered as it describes the data satisfactorily;

- adding the non-resonant components either as wide resonances or as phase-space decay contributions;

- changing the combinatorial background description for the $2 \mathrm{D} m\left(J / \psi, h_{1}=K, h_{2}=\pi\right)$ $-m\left(J / \psi, h_{1}=\pi, h_{2}=K\right)$ distribution from the functional form of Eq. (5.1) to the form of Eq. (5.2);

- changing the combinatorial background descriptions for distributions in the $B^{0}$ and $B_{s}^{0}$ CRs. It is determined by varying the maximal allowed momentum corrections and by using different corrections for two hadron tracks.

The uncertainty of the MC reproducing the hadron track reconstruction resolution is tested by varying the track momentum reconstruction resolution by $\pm 10 \%$ of the actual resolution value. The effect on the resolution after scaling to $m\left(\Lambda_{b}^{0}\right)$ [17] (Section 5) is within $0.1 \mathrm{MeV}$ and thus neglected.

The systematic uncertainties are summarised in Tables 2 and 3. Contributions from the systematic uncertainties listed above are added in quadrature separately for positive and negative variations.

\section{Testing the hypothesis without pentaquarks}

The iterative procedure described in Section 5 is repeated using the $\Lambda_{b}^{0} \rightarrow J / \psi p K^{-}$decay model without pentaquarks. 


\begin{tabular}{|c|c|c|c|c|}
\hline Source & $N\left(P_{c 1}\right)$ & $N\left(P_{c 2}\right)$ & $N\left(P_{c 1}+P_{c 2}\right)$ & $\Delta \phi$ \\
\hline Number of $\Lambda_{b}^{0} \rightarrow J / \psi p K^{-}$decays & $\begin{array}{l}+1.8 \% \\
-0.6\end{array}$ & $\begin{array}{l}+6.6 \% \\
-9.2 \% \\
\end{array}$ & $\begin{array}{l}+1.6 \% \\
-0.8\end{array}$ & $\begin{array}{l}+0.3 \% \\
-0.0\end{array}$ \\
\hline Pentaquark modelling & ${ }_{-0}^{+21} \%$ & $\begin{array}{l}+1 \\
-22\end{array}$ & $+8.7 \%$ & ${ }_{-0.0}^{+1.6 \%}$ \\
\hline Non-pentaquark $\Lambda_{b}^{0} \rightarrow J / \psi p K^{-}$modelling & ${ }_{-2}^{+14} \%$ & $\begin{array}{l}+5 \\
-44\end{array}$ & $\begin{array}{l}+9.2 \% \\
-9.1\end{array}$ & $\begin{array}{l}+3.6 \% \\
-1.6 \%\end{array}$ \\
\hline Combinatorial background & $\begin{array}{l}+0.7 \% \\
-4.0\end{array}$ & $+-5 \%$ & $\begin{array}{l}+4.2 \% \\
-4.8\end{array}$ & $\begin{array}{l}+3.2 \% \\
-0.0\end{array}$ \\
\hline$B$ meson decays modelling & $\begin{array}{l}+13 \% \\
-25\end{array}$ & $+28 \%$ & $\begin{array}{l}+1.6 \% \\
-9.3\end{array}$ & $\begin{array}{l}+0.5 \% \\
-2.1\end{array}$ \\
\hline Total systematic uncertainty & $+28 \%$ & $\begin{array}{l}+35 \% \\
-61\end{array}$ & ${ }_{-15}^{+14} \%$ & $\begin{array}{l}+5.1 \% \\
-2.7\end{array}$ \\
\hline
\end{tabular}

Table 2: Systematic uncertainties for measurements of the pentaquark yields under assumption of no interference effects, the yield of a sum of two pentaquarks and the relative phase between pentaquark amplitudes [10].

\begin{tabular}{|c|c|c|c|c|}
\hline Source & $m\left(P_{c 1}\right)$ & $\Gamma\left(P_{c 1}\right)$ & $m\left(P_{c 2}\right)$ & $\Gamma\left(P_{c 2}\right)$ \\
\hline Number of $\Lambda_{b}^{0} \rightarrow J / \psi p K^{-}$decays & $\begin{array}{l}+0.06 \% \\
-0.03\end{array}$ & $\begin{array}{l}+3.5 \% \\
-2.5\end{array}$ & $\begin{array}{l}+0.07 \% \\
-0.04\end{array}$ & $\begin{array}{l}+7 \% \\
-13 \% \\
\end{array}$ \\
\hline Pentaquark modelling & $\begin{array}{l}+0.6 \% \\
-0.0\end{array}$ & ${ }_{-0}^{+18} \%$ & $\begin{array}{l}+0.2 \% \\
-0.0\end{array}$ & $\begin{array}{l}+0 \\
-33\end{array}$ \\
\hline Non-pentaquark $\Lambda_{b}^{0} \rightarrow J / \psi p K^{-}$modelling & $\begin{array}{l}+0.23 \% \\
-0.05 \%\end{array}$ & $\begin{array}{l}+9.2 \% \\
-1.2\end{array}$ & $\begin{array}{l}+0.24 \% \\
-0.02\end{array}$ & $\begin{array}{l}+2 \\
-62 \% \\
\end{array}$ \\
\hline Combinatorial background & $\begin{array}{l}+0.03 \% \\
-0.15\end{array}$ & $\stackrel{+0}{+11} \%$ & $\begin{array}{l}+0.01 \% \\
-0.17\end{array}$ & $\begin{array}{l}+22 \\
-4\end{array}$ \\
\hline$B$ meson decays modelling & $\begin{array}{l}+0.24 \% \\
-0.00 \%\end{array}$ & $\begin{array}{l}+21 \% \\
-21\end{array}$ & $\begin{array}{l}+0.27 \% \\
-0.14\end{array}$ & $\begin{array}{l}+17 \% \\
-57\end{array}$ \\
\hline Total systematic uncertainty & $\begin{array}{l}+0.7 \% \\
-0.2\end{array}$ & $\begin{array}{l}+30 \% \\
-24\end{array}$ & $\begin{array}{l}+0.4 \% \\
-0.2\end{array}$ & $\begin{array}{l}+28 \% \\
-91\end{array}$ \\
\hline
\end{tabular}

Table 3: Systematic uncertainties for measurements of the pentaquark masses and natural widths [10].

Figure 4 (left) shows the $m(J / \psi p)$ projection of the binned likelihood fit of the $m(J / \psi p)$, $m(J / \psi K)$ and $m(p K)$ distributions for the hypothesis without pentaquarks with the default $\Lambda^{*}$ model. The $\chi^{2}$ fit of the $m(J / \psi p)$ distribution alone, shown in Figure 4 (right), yields $\chi^{2} / N_{\text {dof }}=$ $69.2 / 37$ corresponding to a $p$-value of $1.0 \times 10^{-3}$. 

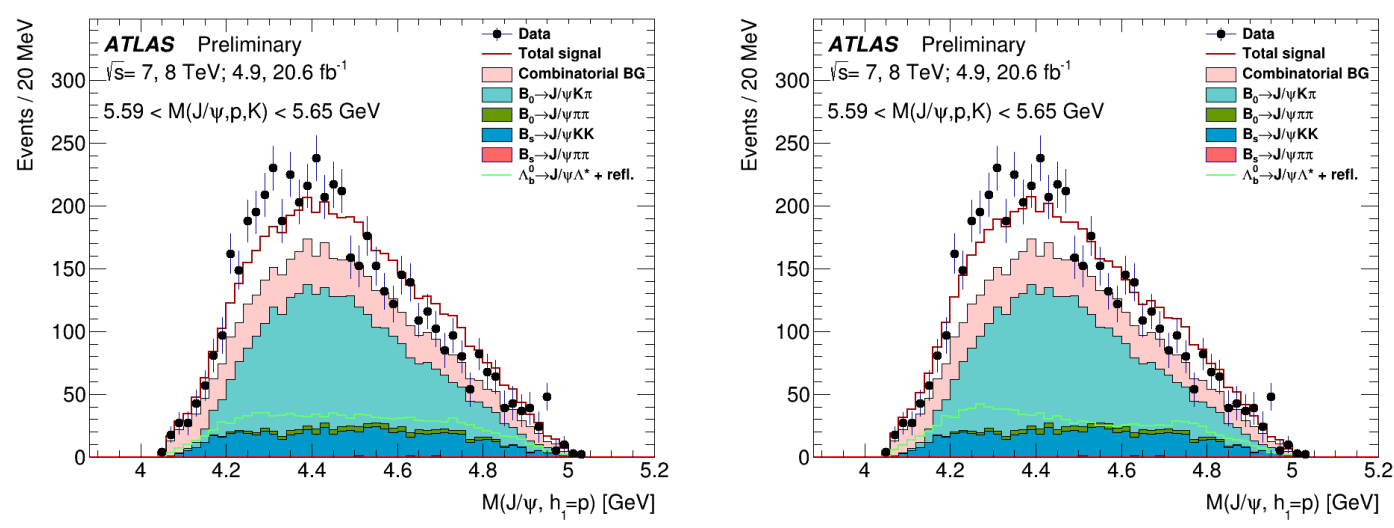

Figure 4: The $m(J / \psi p)$ projection of the binned likelihood fit of the $m(J / \psi p), m(J / \psi K)$ and $m(p K)$ distributions (left) and the $\chi^{2}$ fits of the $m(J / \psi p)$ distribution (right) in the signal region for the hypothesis without pentaquarks with the default $\Lambda_{b}^{0} \rightarrow J / \psi \Lambda^{* 0}$ decay model [10].

All systematic variations described in the previous section and relevant to the hypothesis without pentaquarks are tested. The best data description is obtained using the extended $\Lambda_{b}^{0} \rightarrow J / \psi \Lambda^{* 0}$ decay model. Figure 5 (left) shows the $m(J / \psi p)$ projection of the binned likelihood fit of the $m(J / \psi p), m(J / \psi K)$ and $m(p K)$ distributions for the hypothesis without pentaquarks with the extended $\Lambda_{b}^{0} \rightarrow J / \psi \Lambda^{* 0}$ decay model. The $\chi^{2}$ fit of the $m(J / \psi p)$ distribution alone, shown in Figure 5 (right), yields $\chi^{2} / N_{\text {dof }}=42.0 / 23$ corresponding to a $p$-value ${ }^{4}$ of $9.1 \times 10^{-3}$.
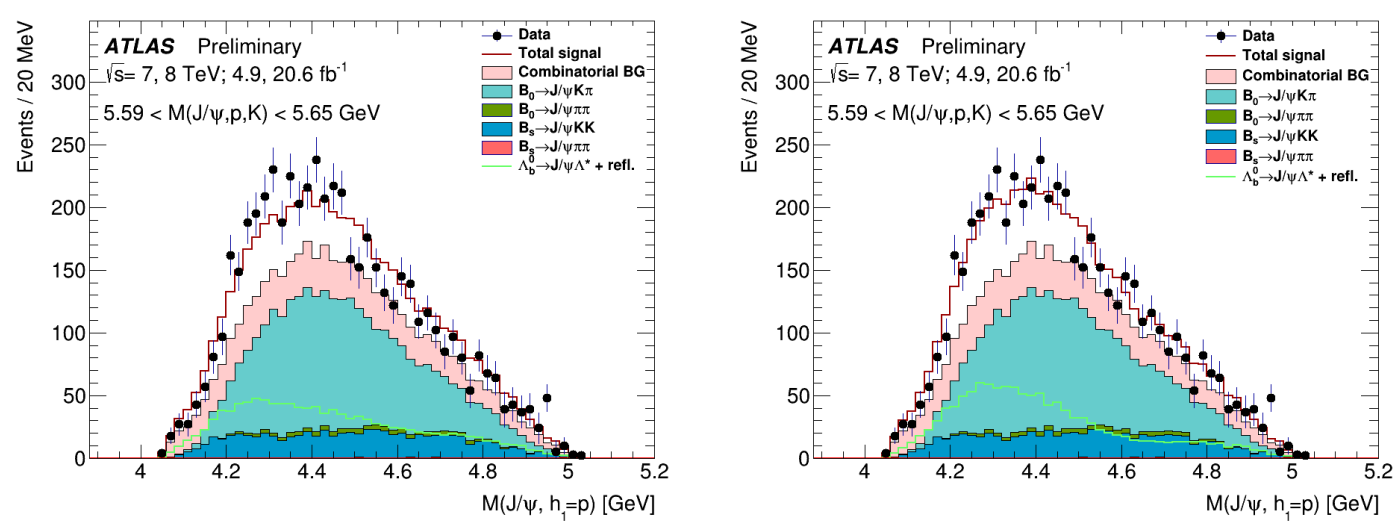

Figure 5: The $m(J / \psi p)$ projection of the binned likelihood fit of the $m(J / \psi p), m(J / \psi K)$ and $m(p K)$ distributions (left) and the $\chi^{2}$ fits of the $m(J / \psi p)$ distribution (right) in the signal region for the hypothesis without pentaquarks with the extended $\Lambda_{b}^{0} \rightarrow J / \psi \Lambda^{* 0}$ decay model [10].

\footnotetext{
${ }^{4}$ The $p$-value is calculated without accounting for effects from combining different systematic uncertainties.
} 

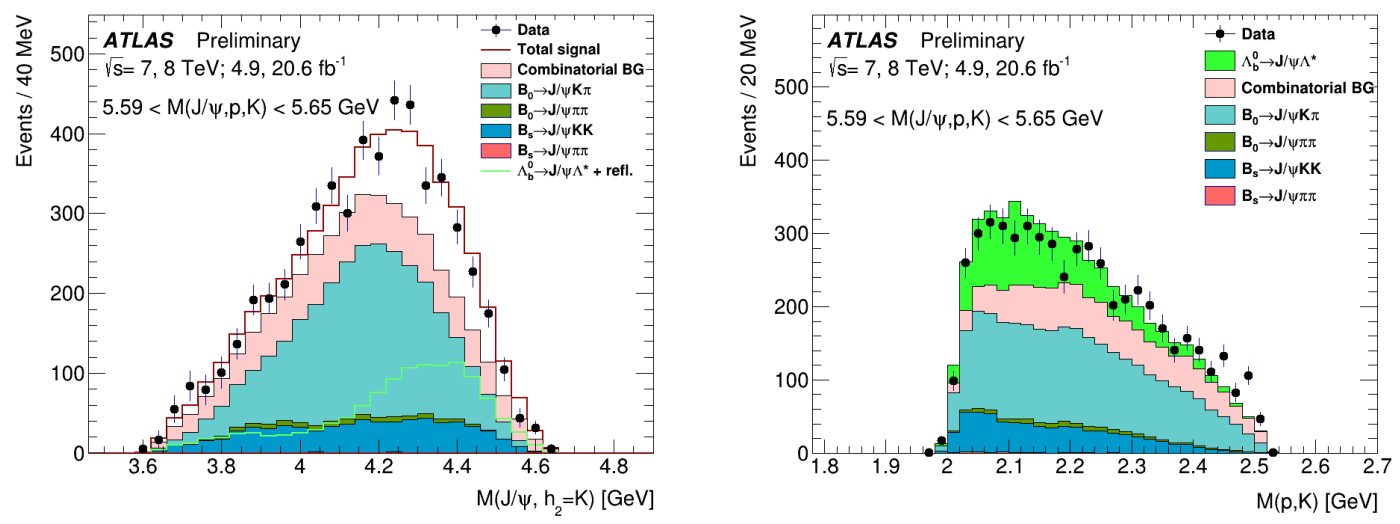

Figure 6: The invariant mass distributions $m(J / \psi K)$ (left) and $m(p K)$ (right) in the signal region overlaid with projections of the $m(J / \psi p)$ fit results for the hypothesis without pentaquarks with the extended $\Lambda_{b}^{0} \rightarrow$ $J / \psi \Lambda^{* 0}$ decay model [10].

Figure 6 shows $m(J / \psi K)$ and $m(p K)$ distributions in the SR overlaid with projections of the $m(J / \psi p)$ fit results for the hypothesis without pentaquarks with the extended $\Lambda_{b}^{0} \rightarrow J / \psi \Lambda^{* 0}$ decay model. Descriptions of all distributions are not so good as for the hypothesis with two pentaquarks though acceptable.

One can conclude that the data prefer the model with two or more pentaquark states. The best result without pentaquarks is achieved with the extended model of $\Lambda_{b} \rightarrow J / \psi, \Lambda^{*}$ decays. The quality of data description with such model is considerably worse in comparison to models with pentaquarks, even if not statistically excluded.

\section{Conclusions}

The analysis of $\Lambda_{b}^{0} \rightarrow J / \psi \Lambda^{* 0}$ decays with large $m\left(p K^{-}\right)$invariant masses has been performed with the ATLAS experimental data on the $p p$ collisions at $\sqrt{s}=7 \mathrm{TeV}$ and $8 \mathrm{TeV}$ at LHC. Hypotheses of no pentaquark signals, two pentaquark signals and four pentaquark signals are considered.

The pentaquark masses and widths obtained using the model with two pentaquarks are consistent with the corresponding LHCb results [5]. The fit of the model with two pentaquarks to data yields $\chi^{2} / N_{\text {dof }}=37.1 / 39$ corresponding to $p$-value $=55.7 \%$. The fit with the two pentaquark signals with their masses and widths fixed to the LHCb values [5] yields $\chi^{2} / N_{\text {dof }}=49.0 / 43$ with $p$-value $=24.5 \%$.

The data are also compatible with the recent LHCb observations of three narrow pentaquarks [9], although do not allow to make independent measurement of their parameters.

Although the data prefer the model with two or more pentaquark states, the model without pentaquarks is not excluded. The $\Lambda_{b}^{0} \rightarrow J / \psi \Lambda^{* 0}$ decay model, considering two lowest orbital momenta between the decay products of $\Lambda^{*}(1800), \Lambda^{*}(1810)$ and $\Lambda^{*}(1890)$, and one lowest orbital momentum between the decay products of $\Lambda^{*}(2100)$ and $\Lambda^{*}(2110)$, describes the $m(J / \psi p)$ distribution yielding $\chi^{2} / N_{\text {dof }}=42.0 / 23$ corresponding to $p$-value $=9.1 \times 10^{-3}$. 


\section{References}

[1] Högaasen, H. and Sorba, P., The systematics of possibly narrow quark states with baryon number one. Nucl. Phys. B 145, (1978) 119

[2] Strottman, D., Multi-quark baryons and the MIT bag model. Phys. Rev. D 20 (1979) 748

[3] Lipkin, H. J., New possibilities for exotic hadrons: Anticharmed strange baryons. Phys. Rev. Lett. B 195 (1987) 484

[4] Hicks, K.-H., On the conundrum of the pentaquark. Eur. Phys. J. H 37 (2012) 1

[5] LHCb Collaboration, Observation of $J / \psi p$ resonances consistent with pentaquark states in $\Lambda_{b}^{0} \rightarrow J / \psi K^{-} p$ decays. Phys. Rev. Lett. 115 (2015) 072001

[6] LHCb Collaboration, Study of the production of $\Lambda_{b}^{0}$ and $\bar{B}^{0}$ hadrons in pp collisions and first measurement of the $\Lambda_{b}^{0} \rightarrow J / \psi p K^{-}$branching fraction. Chin. Phys. C 40 (2016) 011001

[7] LHCb Collaboration, Model-independent evidence for $J / \psi p$ contributions to $\Lambda_{b}^{0} \rightarrow J / \psi p K^{-}$decays. Phys. Rev. Lett. 117 (2016) 082002

[8] LHCb Collaboration, Evidence for exotic hadron contributions to $\Lambda_{b}^{0} \rightarrow J / \psi p \pi^{-}$decays. Phys. Rev. Lett. 117 (2016) 082003

[9] LHCb Collaboration, Observation of a narrow pentaquark state, $P_{c}(4312)^{+}$, and of two-peak structure of the $P_{c}(4450)^{+}$. Phys. Rev. Lett. 122 (2019) 222001

[10] ATLAS Collaboration, Study of $J / \psi p$ resonances in the $\Lambda_{b}^{0} \rightarrow J / \psi p K^{-}$decays in pp collisions at $\sqrt{s}=7$ and $8 \mathrm{TeV}$ with the ATLAS detector. ATLAS-CONF-2019-048 (2019), http://cds.cern.ch/record/2693957

[11] ATLAS Collaboration, The ATLAS Experiment at the CERN Large Hadron Collider. JINST 3 (2008) S08003

[12] ATLAS Collaboration, Improved luminosity determination in pp collisions at $\sqrt{s}=7$ TeV using the ATLAS detector at the LHC. Eur. Phys. J. C 73 (2013) 2518

[13] ATLAS Collaboration, Luminosity determination in pp collisions at $\sqrt{s}=8 T e V$ using the ATLAS detector at the LHC. Eur. Phys. J. C 76 (2016) 653

[14] Belle Collaboration, K. Chilikin et al., Observation of a new charged charmoniumlike state in $\bar{B}^{0} \rightarrow J / \psi K^{-} \pi^{+}$decays. Chin. Phys. D 90 (2014) 112009

[15] ATLAS Collaboration, Performance of the ATLAS Trigger System in 2010. Eur. Phys. J. C 72 (2012) 1849

[16] ATLAS Collaboration, Performance of the ATLAS muon trigger in pp collisions at $\sqrt{s}=8 \mathrm{TeV}$. Eur. Phys. J. C 75 (2015) 120

[17] M. Tanabashi et al. (Particle Data Group), Review of Particle Physics. Phys. Rev. D 98 (2018) 030001

[18] T. Sjöstrand, S. Mrenna, P. Skands, A Brief Introduction to PYTHIA 8.1. Comput. Phys. Commun. 178 (2008) 852

[19] ATLAS Collaboration, The ATLAS Simulation Infrastructure. Eur. Phys. J. C 70 (2010) 823

[20] Agostinelli, S. and others, GEANT4: A Simulation toolkit. Nucl. Instrum. Meth. A 506 (2003) 250-303

[21] Allison, J. and others, Geant4 developments and applications. IEEE Trans. Nucl. Sci. 53 (2006) 270 
[22] Wick, G. C., On the general theory of collisions for particles with spin. Annals Phys. 7 (1959) 404

[23] Chung, S. U., Spin formalisms. CERN 71-08 (1971)

[24] Richman, J. D., An Experimenter's Guide to the Helicity Formalism. CALT 68-1148 (1984) 